\title{
Nanocharacterization and Electrical Properties of Grain Boundaries in Gd/Pr Doubly-Doped Ceria
}

\author{
W. J. Bowman, J. Zhu, P. A. Crozier
}

School for the Engineering of Matter, Transport, and Energy, Arizona State University, Tempe, Arizona 85287-6106

Grain boundaries in doped ceria electrolytes have a deleterious effect on the total ionic conductivity especially at intermediate temperatures $\left(300-550{ }^{\circ} \mathrm{C}\right)[1,2]$. The high resistivity of grain boundaries has been attributed to a space charge double layer which is believed to create a vacancy depletion region emanating from grain interfaces. Other factors may also contribute to high grain boundary resistance. For example, recent high resolution elemental analysis in the transmission electron microscope (TEM) of $20 \%$ Gd-doped ceria $(G D C)$ by our group and others shows significant Gd segregation to grain boundaries yielding enrichment zones of approximately 60 at $\% \mathrm{Gd}$, far exceeding the optimal Gd concentration (10 - 20 at \%) for maximum ionic conductivity.

We have prepared a series of doped ceria electrolytes for characterization of electrical conductivity by AC-impedance spectroscopy, as well as structural and chemical analysis by electron microscopy. Electrolyte preparation for impedance measurements is performed using mixed oxide powders synthesized by spray-drying, a simple and inexpensive powder synthesis method [3]. Nitrate salts of cerium, gadolinium and praseodymium were used to synthesize powders which were then die-pressed in uniaxial compression at $180 \mathrm{MPa}$ in a hardened steel piston/cylinder assembly at room temperature to yield electrolyte discs. Discs were sintered for $18 \mathrm{hrs}$ at $1350{ }^{\circ} \mathrm{C}$ and Pt electrodes were applied for impedance measurements. Following electrical characterization, TEM samples were prepared by sonic cutting, mechanical grinding, polishing, and argon ion milling of the oxide electrolytes. Atomic resolution imaging and nanospectroscopy were performed using a variety of microscopes operating between $200 \mathrm{kV}$ and $400 \mathrm{kV}$.

The compositional homogeneity of the spray-dried electrolytes, as well as the effect of doping on grain size has been investigated for (nominally) $\mathrm{Ce}_{0.8} \mathrm{Gd}_{0.2} \mathrm{O}_{2-}$ (" $\mathrm{GDC}$ "), and $\mathrm{Ce}_{0.85} \mathrm{Gd}_{0.11} \mathrm{Pr}_{0.04} \mathrm{O}_{2-\delta}$ ("GPDC"). High spatial resolution electron energy-loss spectroscopy in a JEOL ARM200F probe corrected scanning TEM (STEM) has been performed to map the distribution of dopant cations in the vicinity of grain boundaries. In an attempt to elucidate the $\mathrm{O}^{2-}$ vacancy environment near grain boundaries, the local bonding of $\mathrm{Ce}^{4+}$ and $\mathrm{O}^{2-}$ have been probed through observation of the $\mathrm{Ce}_{54}$ and $\mathrm{O} \mathrm{K}$ edges in energy-loss spectra.

Figure B shows an annular dark field (ADF) STEM image of a grain boundary in GPDC with the inset 2D EELS spectrum image color map indicating the segregation of dopant cations to the grain boundary. The color map is created from integration of the background-subtracted Ce, Pr and Gd M45 white lines (fig. A). Figure $\mathrm{C}$ illustrates the compositional variation near the grain boundary estimated by k-factor analysis of background-subtracted white line integrated intensities. A distinct $\mathrm{Ce} \mathrm{M}_{4}: \mathrm{M}_{5}$ white line ratio decrease characteristic of the reduction of $\mathrm{Ce}^{4+}$ to $\mathrm{Ce}^{3+}$ was also observed, possibly indicating an increased oxygen vacancy concentration near the grain boundary. The cation segregation zone was measured at full width half maxima (FWHM) to be 1.6 and $2.0 \mathrm{~nm}$ in the GDC and GPDC, respectively. 
The average grain boundary core composition in the GDC was approximately $61 \%$, and the preliminary results presented here indicate grain boundary core compositions of $[\mathrm{Pr}] \approx 16 \%$ and $[\mathrm{Gd}] \approx 29 \%$.

We present results of electrical conductivity measurements and EELS of grain boundaries in ceria doubly doped with Gd and Pr. We discuss correlations between $\mathrm{Ce}^{4+}$ oxidation state variations, dopant segregation and resultant electrical properties in these materials. The lower Gd content and higher $\mathrm{Pr}$ content of the GPDC grain boundary results in much higher ion conductivity.

\section{References}

1. Guo, X. et al, Journal of The Electrochemical Society, 148 (3) E121-E126 (2001)

2. Avila-Paredes, H.J. et al, Solid State Ionics 177 (2006) 3075-3080.

3. Sharma, V. et al, Chemical Physics Letters, 495 (2010) 280-2862.

4. We thank the National Science Foundation's Graduate Research Fellowship Program (DGE1311230), and NSF DMR 1308085 for funding this work. We also gratefully acknowledge support of ASU's John M. Cowley Center for High Resolution Electron Microscopy.
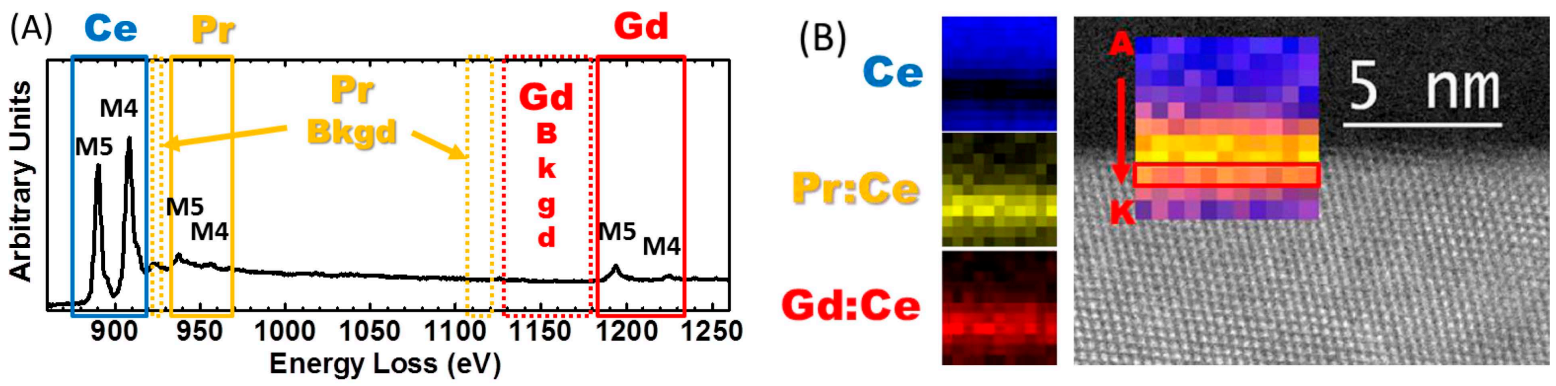

(C)

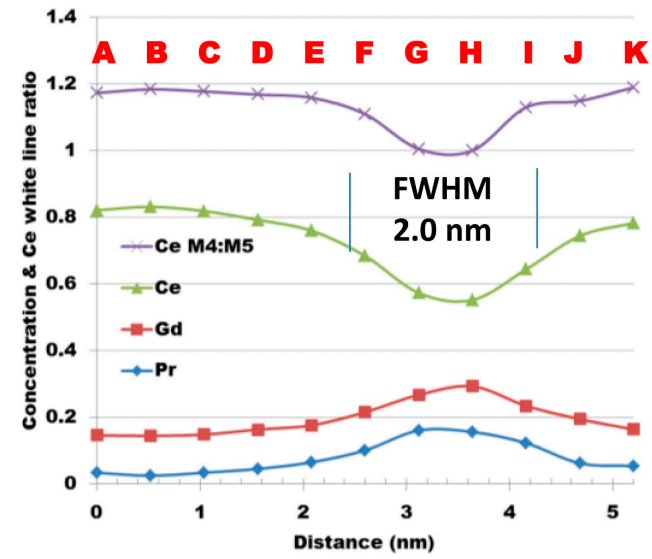

Fig. A. GPDC core loss EELS with highlighted $\mathrm{M}_{45}$ white lines and background fitting windows for Pr and Gd. Ce background window is omitted for clarity.

Fig. B. ADF STEM micrograph of a GPDC grain boundary with inset EELS spectrum image color map created using the integrated intensities of $\mathrm{Ce}$, Pr and $\mathrm{Gd} \mathrm{M}_{45}$ white line in (A).

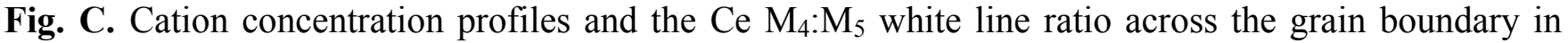
(A); each point is derived from row-by-row signal integration (red box labeled $\mathrm{A} \rightarrow \mathrm{K}$ in (B)). 